\title{
Le Prologue du Franklin's Tale et les Lais bretons.
}

Nous savons aujourd'huj, à n'en pas douter, que le Conte du Franklin, 1 en dépit de l'affirmation de Chaucer, n'est pas un lai breton, c'est-à-dire, n'est pas emprunté aux traditions celtiques. M. Rajna a montré qu'il vient en droite ligne de Boccace qui a traité le même thème deux fois, dans le Filocolo et dans le Décaméron. ${ }^{2}$ Il reste que Chaucer mentionne expressément les Bretons. ${ }^{3}$ Pourquoi ce prologue trompeur, et qui a pu lui en suggérer l'idée? Nous savons par ailleurs que c'est au XIle siècle surtout que les lais narratifs ont fleuri. Le souvenir en était-il encore vivant au XIVe siècle? Devons-nous admettre qu'à l'époque de Chaucer, à côté du sens si souvent attesté de chant, chanson, l'anglais donnait couramment au mot lai le sens de poème narratif sur la matière de Bretagne? Ou ne faut-il voir dans l'emploi qui nous occupe qu'une survivance, un archaïsme artificiellement maintenu? On voudrait, dans l'article qui va suivre, essayer de répondre à ces questions. Ce serait par là-même déterminer, en outre, s'il y a eu parallélisme entre l'histoire du lai français et celle du "lay" anglais, ou si le mot et le genre, transplantés en Angleterre, y ont eu une fortune différente. 4

Que nous dit M. Rajna sur ce point? Reprenant une indication de M. Schofield, 5 il signale l'étroite ressemblance de notre prologue à l'introduction d'Equitan de Marie de France. Mais là où le critique américain voyait simplement analogie - les débuts de tous les lais se ressemblant plus ou moins - M. Rajna va plus loin: il est bien près de déclarer qu'ici Chaucer a imité Marie. En tout cas, suivant lui, Chaucer connaissait les Lais de la poétesse française, et que dans notre prologue il ait eu Equitan sous les

1 Edition Walter W. Skeat, 6 volumes, Oxford, 1894 et un volume de supplément, Oxford, 1897.

2 Romania, XXXII, 204 et s.

3 Thise olde gentil Britons in hir dayes / Of diverse aventures maden layes, | Rymeyd in hir firste Britton tonge; | Which layes with bir instruments they songe, | Or elles redden hem for hir plesaunce; | And oon of hem have $I$ in remembraunce, , Which $I$ shall seyn with good wil as I can. (Canterbury Tales, v. 11021-1 1027.)

4 Pour l'histoire du lai français voir Zeitschr. f. rom. Phil., XXIX, 293-322. On trouvera en outre, aux pp. 312-314, sur l'histoire du lay anglais quelques indications, que le présent article se propose de développer et de compléter.

- Publ. of the Modern Lang. Assoc. of America XVI, 427. 
yeux ou n'ait fait que reproduire une formule courante, il reste que le poète anglais a emprunté les données de son conte à Boccace et les a modifiées habilement ici et là, pour en faire un lai à la manière de Marie: il rendait là, en reproduisant une forme de composition chère aux Celtes, une espèce d'hommage d̀ une race qui formait une partie constitutive de la nation, ou tout au moins de l'Etat anglais. Le prologue traditionnel dans le lai breton suivait tout naturellement de cette transformation de la nouvelle italienne. ${ }^{1}$ Il est assurément dangereux, en pareille matière, de se montrer trop affirmatif, mais on peut dire que l'explication de M. Rajna a bien des chances d'être la vraie. Sur un point cependant, elle reste douteuse ou incomplète. Il est évident que Chaucer tenait de quelqu'un son idée du "lai breton", mais est-il aussi sûr que ce soit de Marie? Est-il vraisemblable que l'auteur du Conte du Franklin ait connu l'auteur d'Equitan?

Notons d'abord que l'on ne trouve nulle part dans le reste de l'œuvre des traces d'une semblable influence. Le conte de la Femme de Bath nous reporte, il est vrai, à la cour du roi Arthur, et, suivant Gaston Paris, ${ }^{2}$ serait fondé sur un lai français. Mais ce n'est là qu'une hypothèse, et de recherches récentes il semble résulter qu'en tout cas le conte de Chaucer ne remonte au lai qu'à travers un ou plusieurs intermédiaires. ${ }^{3}$ S'il y a eu influence de Marie, c'est dans le conte du Franklin, et là seulement, qu'elle s'est exercke. 4 On lui a emprunté quelques renseignements sur les lais et la conception même, le type d'un certain genre littéraire. Que l'imitation se soit bornée là, et que Chaucer connaissant les Lais ne se soit soucié d'en faire passer aucun en anglais, voilà qui est déjà surprenant; mais enfin cela n'est pas impossible. Voyons si c'est probable.

Chaucer assurément connaissait bien notre langue et une partie notable de la littérature française lui était très familière. Il est aujourd'hui facile, après tant de pénétrantes recherches qui lui ont été consacrées, d'énumérer les auteurs qu'il a connus. ${ }^{5}$ Comme il

1 Romania, art. cit., $23 \mathrm{I}-233$.

- Hist, litt. de la France, XXX, 102.

3 Voir G. H. Maynadier, The Wife of Bath's Tale, its Sources and Analogues, London, 1902, et cf. le compte rendu de M. Koch dans Englische Studien, XXX, 460-64 et la note de M. Rajna, Romunia, XXXII, 233. n. I. - Du reste que signifie le mot 'lai' ici?

4 Si des Lais nous passons aux Fables, les points de contact sont-ils plus nombreux? Il ne le semble pas. On rapprochait volontiers autrefois le Nun's Priest's Tale d'une fable de Marie; mais nous savons aujourd'hui qu'en réalité le conte de Chaucer - en tant qu'il se rapporte aux aventures de Chantecler et de Renard - a sa source immédiate dans quelque conte épique appartenant au cycle de Renard. On a méme pu déterminer avec assez d'exactitude la position de l'original de Chaucer par rapport au Reinhart Fuchs allemand et a la Branche II du Renard français. Voir K. O. Petersen, On the sources of the Nonne Prestes Tale, Boston, 1898.

5 Pour les travaux antérieurs à 1892, voir les résultats obtenus dans T. R. Lounsbury, Studies in Chancer, New York, 1892, t. II, p. 206-223 et 
est naturel, il se montre très au courant des cuvres contemporaines ou récentes. Il imite Machaut, dont se réclament tous les poètes du XIV' siècle, en plus d'un endroit; il imite aussi à l'occasion Froissart, avec qui il s'est peut-être rencontré d̀ Londres; avec Deschamps, il est en commerce littéraire suivi, et il lui doit maint vers et mainte suggestion $;^{1}$ enfin il traduit assez librement trois ballades d'Oto de Granson, "fleur de ceux qui écrivent en France". Ajoutons à cela quelques emprunts à des gens moins connus, Guillaume de Digulleville et Nicolas Trivet. Voilà pour le XIVe siècle: en réalité, toutes ces imitations se ramènent d̀ peu de chose. Envers le XIII' siècle, ses obligations sont plus grandes: il doit le sujet d'un de ses contes au Roman du Renard; 2 mais c'est surtout au Roman de la Rose qu'il est redevable. Il l'avait traduit tout entier, et peut-être a-t-on conservé un fragment de cette traduction. En tout cas, c'est le seul ouvrage français qui ait réellement exercé sur lui une influence durable et profonde: toute sa vie, il est resté, comme ses contemporains de France, sous le charme de cette cuvre maîtresse du XIIIe siècle. Nous ne voyons pas que Chaucer soit remonté plus haut dans ses lectures: s'il est au courant de la littérature latine du XII ${ }^{e}$ siècle, ${ }^{3}$ la littérature française de la même époque semble lui être restée inconnue. Et pourquoi en serions-nous surpris? Qui donc parmi ses amis de France la connaissait davantage? C'est le moment où une nouvelle littérature se lève et une nouvelle conception de la poésie se fait jour. ${ }^{4}$ Entre Machaut et ses successeurs et les poètes de l'âge précédent, il y a peu de points de contact: il y a eu rupture de la tradition poétique. Du passé, il ne survit guère, parmi les grandes ceuvres que le Roman du Renard, dont l'évolution du reste touche alors d sa fin, et surtout le Roman de la Rose, dont l'influence se fera sentir jusqu'au milieu du $\mathrm{XVI}^{e}$ siècle. Le reste est mort. En particulier, il y a lieu de croire qu'on a complètement oublié Marie. ${ }^{6}$ Des trois mss. fran-

t. III, p. 450-51. Parmi les travaux postérieurs, citons G. L. Kittredge, Engl. Studien, XXVI, 321-336, Modern Philology, I, I et s., K. O, Petersen, ouvr. cit., et Publ. of Mod. Lang. Assoc., XI, 173 et s.; W. E. Mead, Publ. of Mod. Lang. Assoc., XVI, 388 et s.; H. S. V. Jones, ibid., $\mathrm{XX}, 346$ et s. Cf. la note suivante.

1 Jusqu'à ces derniers temps on n'avait pas réussi à découvrir chez Chaucer le moindre emprunt a Deschamps. Mais dans un article récent $M$. J. L. Lowes en a signalé d'indéniables, Publ. of Mod. Lang. Assoc. of America, $\mathrm{XIX}, 593$ et $\mathrm{s}$.

Voir note 4 S. 699.

- Voir Lounsbury, Studies in Chaucer, t. II, p. $30 \mathrm{r}$ et s. et spécialement p. 418.

4 Voir G. Paris, La littérature française au XIV e siècle, dans la Poésie du Moyen-Age, 2 ème Série, p. 185.

- Il serait curieux que son souvenir se fat mieux conservé en Espagne, et c'est pourtant ce qui est arrivé, si l'on en croit M. Fitzmaurice Kelly: selon lui, l'auteur de El Cavallero Cifar a connu les Lais de Marie. (Littérature Espagnole, Paris, 1904, pag. 68-69). Mais c'est ce qui ne semble 
çais 1 qui ont conservẻ ses œuvres, deux datent du milieu ou de la fin du XIIIe siècle: le ms. I IO4 (qui contient neuf lais de Marie, plus quinze autres d'auteurs divers) et le ms. 2 I68 (qui contient trois lais de Marie). Seul le ms. 24432 ne remonte pas plus haut que 1332 : il ne renferme du reste que Yonec, et le scribe ne se doute nullement que le conte qu'il copie fait partie d'une collection plus étendue. ${ }^{2}$ Mais il $y$ a une preuve plus frappante et plus sûre de l'oubli dans lequel Marie est tombée. Le XIV' siècle ne sait plus ce que c'est qu'un "lai breton", au sens où l'on applique ce mot à Guigemar ou Lanval par exemple; le mot lai est fréquent chez les poètes, mais toujours à cette époque, il désigne un chant (souvent une plainte amoureuse - ou encore le chant des oiseaux) ou plus particulièrement un genre lyrique spécial soumis à des règles fixes. Machaut, Froissart, Deschamps ne connaissent pas d'autre sens, et dans son Art de Dictier le dernier donne les règles du lai lyrique de douze stances comme il donne celle de la ballade et du virelai. Même au XIII ${ }^{e}$ siècle, l'autre sens du mot (poème narratif), timidement introduit au XII ${ }^{e}$ par Marie de France, n'a pas prévalu pendant bien longtemps, ni dans un cercle très étendu. Quelques compositions de cette époque seulement, rattachées avec plus ou moins de droit à la matière de Bretagne, s'intitulent lais. A leur exemple, des poèmes narratifs, qui n'ont rien d̀ voir avec la Bretagne et sont en réalité des fabliaux, sont décorés du nom de lai. Mais cet usage a été très restreint et il disparaît dans le premier quart du XIV ${ }^{\text {e }}$ siècle. ${ }^{3}$

Nous arrivons ainsi à deux conclusions importantes pour nous: $1^{0} \mathrm{Ce}$ ne sont pas les Français du XIVe siècle qui pouvaient initier

pas absolument démontré. Certainement le roman espagnol - outre certaines ressemblances, assez vagues du reste, avec des passages d'Eliduc, de Guigemar et de Doon - renferme une allusion tres nette a l'histoire de Lanval ou de Graelent. (Voir le passage cité dans $\mathrm{Ch}$. $\mathrm{Ph}$. Wagner, The sources of El Cavallero Cifar, Revue Hispanique, 1903, p. 50). Mais des recherches et des rapprochements de $M$. Wagner il semble bien résulter que la source directe de l'auteur espagnol était un conte français où les divers éléments que l'on retrouve dans l'histoire de "l'Empereur qui ne riait jamais" étaient déjà réunis et fondus. (Cf. Wagner, art. cit. p. 51): en particulier la confusion entre l'histoire de Lanval et celle d'Yvain devait déjà s'être produite dans cet original français. M. Wagner remarque que dans Lanval le nom de la reine Guinevere n'est pas mentionné (p. 5I); mais il ne l'est pas davantage dans Graelent: autre addition qui doit être du fait du modele immédiat de l'auteur espagnol. El Cavallero Cifar date des dernières années du XIII e siècle ou des toutes premiéres du XIVe siècle. Sa source française - pour le passage qui nous intéresse - nous renvoie donc en pleín XIII e siécle, à un moment où les Lais narratífs étaient encore en faveur: l'auteur du roman espagnol n'a pas connu directement les œuvres de Marie ou de ses imitateurs immédiats. Notons à titre de renseignement, qu'il ne mentionne pas le mot 'lai'.

1 Sur ces manuscrits, voir Warnke, Die Lais der Marie de France?, XXXVIII-IX.

"Le vers I de Yonec: "Puis que des lais ai comenciéc" devient chez lui : "Puis que du lai ....". "Warnke, Lais", p. 123.

"Voir Zeitschr.f. rom. Phil., XXIX, art. cit., en particulier 310-312. 
Chaucer à la connaissance de Marie: ils Pignoraient eux-mêmes profondément; $2^{\circ}$ Ce n'est pas la littérature française du XIVe siècle, ni même celles des œuvres du XIII' siècle qu'il connaissait, qui pouvaient lui fournir le sens qu'il donne au mot 'lai' dans son Prologue du Franklin's Tale: ce sens était devenu complètement étranger à la langue. - Si nous devons trouver une solution aux questions que nous nous sommes posées, clairement c'est hors de France qu'il faut la chercher.

Abandonnant Marie pour un moment, demandons-nous si ce sens du mot lai, inconnu au français du XIVe siècle, est au moins fréquent dans l'usage de Chaucer lui-même. Passons en revue les dix exemples du mot relevés par le lexique de l'édition Skeat. 1 Il faut tout d'abord noter un emploi du mot dans son sens technique de genre lyrique spécial d forme fixe: Aurelius se lamente que son amour ne soit pas payé de retour:

of swich matere made he manye layes,

Songes, compleintes, roundels, virelayes.2

Il y a là une énumération des genres poétiques chers au XIVe siècle qu'on trouve souvent chez les poètes français contemporains: 'lai' y rime généralement avec 'virelai'. 3 Le Prologue de la Ligende des Femmes vertueuses nous offre un exemple analogue; la Reine d'amour défend Chaucer auprès du Dieu d'amour qui l'accuse d'avoir écrit la Rose et Troïlus:

(Version B) 414 Al be hit that he can nat wal endyte,

Yet hath he maked lewed folk delyte

To serve you, in preysing of your name.

He made the book that hight the Hous of Fame...

422 And many an ympne for your halydayes,

That highten Balades, Roundels, Virelayes; ...

430 He hath mad many a lay and many a thing."

Il faut néanmoins remarquer que le 'lai' est ici séparé par huit vers des 'balades', 'roundels' et 'virelayes': le vers qui contient le mot termine le passage et en est comme une espèce de résumé. Lai est donc ici un terme général qui désigne toute espèce de court poème lyrique sur un thème amoureux. C'est bien du reste ainsi que Chaucer emploie le mot le plus souvent: pour lui, il est synonyme de complaint avec lequel il l'accole fréquemment. La Complainte de Vénus traduite de Granson se termine par le vers; "Thus wol I ende this compleynt or lay".5 Citons

1 Tome VI.

2 Frankeleyns Tale, 947-948.

- Cf. par exemple ces vers de Machaut: „... faisiés balades, | Rondeaus, motés et virelais, | Complaintes et amoureus lais" cités par M. Schofield, art. cit. , 445 .

- Cf. version A, v. 402 et s.

- The Compleynt of Venus, v. 71. C'est une série de trois ballades, terminée par un envoi. Il est a remarquer que l'original français n'emploie pas le mot lai. Voir le texte de Granson dans Romania, XIX, 411-416. 
encore deux autres exemples analogues: ${ }_{n}$ In manere of a compleynt

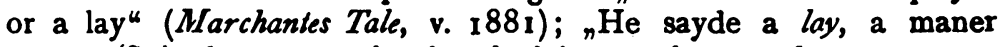
song. (Suit deux vers plus bas le lai, une chanson de onze vers. Puis Chaucer reprend:) Whan he had mad thus his complaynte..." (Book of the Duchesse, v. 47 I). Dans Sir Thopas, le mot est appliqué au chant des oiseaux, sens fréquent en français depuis le XIle siècle: "The thrustelcok make eek his lay" (v. 1959). Nous trouvons trois autres exemples de cet emploi. Mais là encore Chaucer pense plutôt au sentiment, à l'inspiration du chant qu'au rythme et à la mélodie : les oiseaux au temps des amours épanchent, eux aussi, leurs 'complaintes': ce qu'ils chantent, c'est un lai d'amour. (Layes of love, Rose, v. 7 15, Leg. of Good Women, B. v. I40; a lay of love, Troil. and Cr., v. 921). Il ne reste plus qu'un exemple à considérer, c'est précisément celui que nous offre le Prologue du Conte du Franklin:1 là - et là seulement - le mot 'lai' signifie poème narratif sur un sujet se rapportant à la matière de Bretagne: Chaucer nous dit expressément qu'il va nous raconter un 'lai' breton.

Ainsi, en règle générale, Chaucer, comme on pouvait s'y attendre, donne au mot 'lai' le sens qui était courant dans la poésie française contemporaine. Dans un cas, et un cas seulement, il lui attribue un sens insolite dans la langue de Machaut et de Froissart. D'où tient-il cet emploi, et l'a-t-il donc emprunté à quelques-uns de ses devanciers anglais?

Le mot 'lai' n'apparaît pas en Angleterre avant le XUI' siécle. Le plus ancien exemple qu'on en ait découvert jusqu'd̀ présent date d'environ 1240: il se trouve dans l'Ureisun of Ure Lefdi, court poème publié par Morris dans la collection des Old English Homilies. ${ }^{2}$ C'est un hymne en l'honneur de la Vierge, et le moine qui l'a composé exprime vers la fin l'espoir que ses amis retireront quelque profit de ce qu'il a chanté d̀ Marie ce 'lai' anglais, „pet ich habbe i-sungen pe tesne englissce 'lai' ". Il s'agit donc ici d'un chant. C'est là le sens original du mot en français et il n'est pas surprenant de trouver que c'est aussi, chronologiquement, le premier sens attesté en anglais. Le plus ancien exemple, après celui de l'Ureisun, nous amène au début du XIVe siécle:

52ro Mery time it is in May,

The foules syngeth ber lay. ${ }^{3}$

1 Le mot revient deux fois, a deux vers de distance, ce qui nous donne nos dix exemples. "Thise olde gentil Britons ... maden layes, rymeyed ... which layes ... they songe." (C. T., v. 710, 712.)

Londres, 1868. (Early English Text Society.) - Morris suggère (Préface, p. VIII) que le 'lai' anglais pourrait bien être une traduction du latin. $\mathrm{Si}$ nous avons réellement affaire ici à une traduction, on pourrait aussi penser a un original français. Les chants consacrés à la Vierge sont nombreux, comme on sait, au XIII e siècle, et, à côté des lais lyriques profanes de cette époque, nous avons conservé plus d'un lai lyrique religieux.

3 King Alisaunder, dans Weber, Metrical Romances, 1810, Vol. I. 
Le mot signifie ici chant des oiseaux, ce qui est aussi un emploi très fréquent en français. Le Sir Tristrem, vers 1320, nous offre plusieurs exemples du mot 'lai', et c'est toujours au sens de chant ou chanson: 1 l'original français, le Tristan de Thomas n'en connaît en effet pas d'autre. Avec Sir Orfeo ${ }^{2}$ (composé à peu prés à la même époque), nous rencontrons pour la première fois l'autre sens du mot: l'auteur n'appelle pas expressément son poème un 'lai', mais dans un prologue où il cherche d̀ nous renseigner sur les lais bretons, il nous donne des illustrations qui nous renvoient, sans le moindre doute, aux lais narratifs français. Le poème vaut donc la peine que nous nous y arrêtions un moment. ${ }^{3}$ C'est une traduction d'un original français, aujourd'hui perdu, où la légende d'Orphée, telle que la rapporte Ovide, habilement transposée à la mode celtique, était devenue un conte de fée. Le lai d'Orphíe a dû être un des derniers composés des lais français: non seulement l'auteur emprunte dans son prologue son érudition d̀ Marie, mais il a l'air de connaître toute la série de ses imitateurs. Ce prologue a comme un air de famille avec ceux de Tyolet et de Doon: il est en effet composé suivant les mêmes procédés et témoigne du même état d'esprit. D'un côté comme de l'autre, on fait parade, sur les Bretons et leurs coutumes, d'une science qui ne remonte pas plus haut que le texte de Marie: d'un côté comme de l'autre, on n'est pas toujours sûr d'avoir compris la poétesse. Qu'appelle-t-elle au juste un lai? Tantôt, et le plus souvent, elle réserve ce mot pour une composition bretonne, très distincte de son conte, tantôt elle semble bien en désigner ce conte lui-même. La vérité est qu'avant Marie le mot 'lai' ne signifiait que chanson et que c'est elle qui l'a, pour la première fois, employé dans l'autre sens: mais elle ne s'est avisée de ce néologisme qu'assez tard, et dans ses derniers lais. C'est ce que ne virent pas très bien ses imitateurs, et en présence d'apparentes contradictions, ils restèrent perplexes. Les uns, à l'exemple du Guigemar de Marie, continuèrent à distinguer soigneusement entre le conte français et le lai breton; d'autres, qui auraient pu, eux aussi, citer leurs textes, employèrent les deux mots comme synonymes." L'auteur d'Orphee, pendant une grande partie de son prologue, n'a garde de faire cette confusion, et ce n'est pas un 'lai', mais bien une 'aventure' qu'il va nous conter: mais cela ne l'empêche pas, voulant nous donner des exemples de lais, de nous renvoyer - et pour cause - non aux Bretons, mais à Ignaure, à Nabaret, au Lecheor, c'est-à-dire à des contes faits pour la lecture, non à des chansons de harpe. Voilà donc qu'indirectement - par la traduction d'Orphée - un néologisme créé par Marie entre dans la langue anglaise.

1 Ed. Eugen Kölbing, Heilbronn, 1882.

- Ed. O. Zielke, Breslau, 1880.

- Sur le rôle et l'importance du Sir Orfeo dans l'histoire du lai en France et en Angleterre, voir Modern Language Notes, 1906, p. 46 et s.

- Voir Zeitschr. f. rom. Phil., art. cit., 3II. 
Voyons si les œuvres mêmes de Marie n'ont pas pu contribuer directement au même résultat. Ses contes ont été écrits en Angleterre et nous savons par Denis Pyramus qu'ils y ont été très populaires. 1 Pendant longtemps, on dut les lire en français, les gens cultivés ne parlant pas d'autre langue. Les deux seuls manuscrits anglo-normands des Lais qui nous soient restés ${ }^{2}$ datent, l'un du milieu du XIIIe siècle, l'autre de la fin du même siècle ou du commencement du siècle suivant. C'est vers le début du $\mathrm{XIV}$ e siècle que, l'anglais prenant décidément le dessus, on dut se mettre à traduire Marie. Nous ne savons si la collection complète de ses contes passa ainsi en anglais. En tout cas, nous n'avons la traduction que de deux d'entre eux: le Fraisne et Lanval. ${ }^{3}$

1 Vie de St Edmund, éd. Ravenel, Philadelphie, 1906, จ. 35-48.

2 Warnke, Lais' ${ }^{2}$ XXXVIII-IX (Manuscrits H et C).

3 Notez que si le ms. H, milieu du XIII siècle, contient le Prologue, qui ne se retrouve nulle part ailleurs, et douze lais, le ms. $C$, en revanche, qui est au moins de 50 ans postérieur, ne contient plus que Lanval, qui est précisément un des deux lais dont nous avons une traduclion anglaise. Et le conte y est bien nettement détaché du reste de la collection: le vers I : "L'aventure d'un altre lai ... [ [vus cunterai]" y est devenu: „L'aventure de vn lay ..." - Peut-être pourrait-on trouver quelques traces d'autres versions anglaises de lais français. M. Kittredge a démontré que le poème de Chestre, dont nous disons un mot un peu plus loin, est fondé d'une part sur une traduction anglaise de Lanval (perdue sous sa forme originale, mais dont il nous reste plusieurs remaniements), d'autre par sur le lai de Graelent (Americ. Fourn. of Philology, X, I et s. Cf. Schofield, Publ. of Mod, Lang. Assoc., $\mathrm{XV},{ }_{15} \mathrm{I}$ et s.). En ce qui concerne cette seconde imitation, comment fautil se représenter les choses? Chestre avait.il sous les yeux le lai français luimême, ou, comme il semble plus naturel, se servait-il d'une traduction anglaise dans les deux cas? Si l'on adopte cette seconde hypothèse, il faudrait mentionner, à côté de Landaval et du Lay le Freine, un Graelent anglais. Ce qui complique la question, c'est qu'on ne sait pas au juste à quelle époque a vécu Chestre et que des opinions assez différentes ont été émises à ce sujet. - Si l'on admet que le 'lai breton' auquel se référe l'auteur de Sir Gowther est Tydorel, (voir plus loin page 708), la méme question se pose: s'agit-il du Tydorel français que nous connaissons (qui n'est lui-meme qu'un remaniement d'un lai antérieur), ou d'une traduction anglaise? - Faut-il voir une allusion au Yonec de Marie dans un passage du Cursor Mundi - dont je dois l'indication à mon collègue M. Carleton F. Brown - où l'auteur, énumérant les romans qui étaient populaires de son temps, cite un certain 'Ioneck' ('Ionek' suivant un second ms., 'king Ionet' suivant un troisieme)? Il y est question "[of] Tristrem and bys leif Ýsote ... | O Ioneck and of Ysambrase | O Ydoine and of Amadase." (Ed. R. Morris, Early English Text Society, Vol. I, v. 17, 19, 20). Il faut noter qu'un quatrième ms., également reproduit dans l'édition Morris, donne, pour le vers 19: "Of king Ion and of Isombras". C'est peutêtre là la bonne leçon; en tout cas, c'est celle qui est acceptée par l'éditeur: voir le sommaire du poème (Vol. I, Préface, p. VII): ,... of Tristram and his love Isoude, of Isumbras and Iohn, of Amadas and Idoyne. "Voir aussi à l'Index of Names', dressé par Max Kaluza (vol. III), l'article suivant: „Ioneck (Ionek; Ionet; Ion): Joneck, Fohn." Il resterait néanmoins que certains scribes semblent avoir eu connaissance d'une forme Ioneck ou lonek: cette forme leur a-t-elle été suggérée par le Yonec de Marie qu'ils connaissaient soit sous sa forme française soit dans une imitation anglaise? Il est difficile de le décider. - Enfin, on a souvent voulu voir dans le poème intitulé Chaucer's Dream (ou mieux The Isle of Ladies) - qui est probablement du XV e siècle 
Le Lay le Freine, ${ }^{1}$ quị doit avoir été écrit à peu près en même temps que Sir Orfeo, emprunte à celui-ci son prologue et y coud deux vers qui sont un écho des deux premiers vers du Fraisne français :

2 Ac herkneth, lordinges, sothe to sain, Ichil zou telle Lay le Frayn.?

Voild encore notre néologisme qui reparaît, et ici provenant directement de Marie. Même emploi plus loin:

229 And for it was in an asche yfounde,

She cleped it Frain in pat stounde.

pe freyns [name?] of pe asche is a freyn

After pe language of Breteyn.

233 Forpi le Frein men clepep pis lay,

More pan asche in ich cuntray.

Lanval fut vraisemblablement traduit à la même époque. C'est sur cette traduction, qui ne nous est du reste conservée que dans des remaniements, ${ }^{3}$ que se fonde le Launfal Miles de Thomas Chestre. ${ }^{4}$ Chose curieuse, les remaniements laissent tomber les vers où le mot 'lai' était employé. Seul, Chestre le conserve au début de son poème:

I Be douzty Artours dawes, pat held Engelond yn good lawes,

per fell a wondyr cas

4 Of a ley pat was ysette, pat hyzt Launual and hatte zette.

Now herkenep how hyt was. 5

Ainsi, avant Chaucer, le sens premier et original du mot 'lai', en anglais comme en français, est chanson; mais, en anglais comme en français, l'exemple de Marie a fait entrer un nouveau sens dans la langue, celui de poème narratif sur un sujet se rapportant à la

- des imitations directes de Guigemar et d'Eliduc. Depuis longtemps H. Morley a fait justice de ces precendus rapprochements (English Writers, II, 1 , London, 1867, p. 213). Mais $M$. Skeat (dans le VII e volume de son Chaucer, 1897 , p. XV) semble bien encore accepter cette opinion, au moins en ce qui concerne Eliduc; et il ajoute meme Lanval. Il faut redire que, s'il y a sans doute certains traits communs a quelques lais de Marie et au poème anglais du XVe siècle, comme du reste à bien d'autres productions du moyen-âge, il n'y a absolument rien qui nous autorise à ćlablir entre les premiers et le second une relation directe ou même indirecte.

1 Publié par Varnhagen, Anglia, III, 415-425.

2 Marie: "Le lai del Fraisne vus dirai| sulunc le cunte que jeo sai."

${ }^{8}$ Sur le Lanval en Angleterre et toutes les questions qui s'y rattachent, voir Kittredge, American Fournal of Philology, X, I et s.

4 Le poème de Chestre n'a pas été imprimé moins de sept fois. Voir la liste de ces éditions dans Kittredge, art. cit., 2. Je me suis servi de Ritson, Ancient Engleish Metrical Romances, 1802, t. I.

o Cf. Kittredge, art. cit., 14-15. 
matière de Bretagne. Ce sens, introduit plus tard en Angleterre qu'en France, s'y maintient aussi plus longtemps. En effet, dès avant le commencement du XIVe siècle, l'infuence de Marie et de ses imitateurs, qu'on ne lit plus, a cessé de se faire sentir en France: en Angleterre, la substitution de l'anglais au français comme langue du public cultivé a eu pour effet de préserver un certain nombre d'œuvres, celles qui étaient en faveur au moment de cette substitution et qu'on fit, en conséquence, passer en anglais. Alors que Fraisne, Lanval et même Orphée, écrits dans une langue déjà passablement archaïque, tombent, en France, dans un oubli de plus en plus profond, le Lay le Fraine, Landavall et Sir Orfeo, vêtus à la dernière mode anglaise, séduisent encore les lecteurs d'outre Manche.

Parmi ces lecteurs, il faut certainement compter, dans la deuxième moitié du siècle, Chaucer lui-même. Il était familier avec la littérature anglaise de son époque, un passage bien connu de son Sir Thopas le prouve de reste. ${ }^{1}$ En particulier, il a de lire Sir Orfeo qui semble être resté assez longtemps populaire: nous en avons trois manuscrits, dont l'un remonte jusqu'au premier quart du XIVe siècle; ${ }^{2}$ de plus, comme nous l'avons vu, il fournit son prologue au Lay le Freine, dont l'auteur avait dú juger la courte introduction de Marie insuffisante à présenter le conte au lecteur anglais. C'est à ce prologue que Chaucer a dû emprunter les renseignements sur les Bretons qu'il nous redonne au début de son Franklin's Tale: vers 1380 , il ne pouvait guère les puiser ailleurs; nous retrouvons naturellement chez lui la même indécision sur la valeur précise du mot 'lai'; mais il se tire ingénieusement d'affaire en affirmant que les lais, œuvre des anciens Bretons, étaient lantót des chants de harpe, tantôt des récits faits pour la lecture. ${ }^{3}$ C'est probablement encore Sir Orfeo qui a dù donner d Chaucer l'idée de composer, lui aussi, un lai breton. Et la recette ne lui échappa pas: il vit clairement que toute histoire, tout conte pouvait se transformer en un lai breton: il n'y fallait qu'un prologue et un peu de dextérité. 4 ll s'en tira fort bien. Le conte du Franklin ne vient pas des Celtes: mais c'est un „lai breton“ tout de même. ${ }^{5}$ - Ainsi Chaucer n'a pas connu Marie, si ce n'est

1 V. 186-189 et 205. Cf. Lounsbury, ouvr. cit., t. II, p. 201. - Pourquoi n'y a-t-il dans ce passage nulle allusion a Sir Orfeo? Sans doute parce que Chaucer n'y nomme que les romances dont il veut se moquer, en en parodiant plaisamment le style et les procédés.

2 Voir Zielke, ouvr. cit., 23.

3 "... Which layes with hir instruments they songe, | Or elles redden hem for hir plesaunce."

- Par exemple, on place la scéne du récit en pays breton et on donne aux personnages des noms bretons. Cf. Rajna, Romania, art. cit., 233-34.

- Il n'y a pas de contradiction entre ce qui est avancé ici et ce qui est dit dans la première phrase de cet article. Si en employant l'expression 'lai', on pense surtout à un emprunt direct aux légendes celtiques, nous ne connaissons aucun lai breton; si l'on implique simplement une origine celtique, 
peut-être dans les traductions isolées de quelques-uns de ses lais: mais, par l'intermédiaire de Sir Orfeo, il en a subi appréciablement l'influence. De Guigemar au conte du Franklin, en passant par les Deux Amants, Doon, Tyolet et même Ignaure, ${ }^{1}$ il y a une chaîne ininterrompue: curieuse évolution qu'il est piquant de clore ainsi par le grand nom de Chaucer.

Il n'est pourtant pas le dernier qui nous ait, en Angleterre, parlé des lais bretons. Trois poèmes postérieurs au moins portent encore ce titre, Emare, Sir Gowther et le Comte de Toulouse. Emare, ${ }^{2}$ traduction ou imitation d'un poème français auquel l'auteur se réfère souvent, nous affirme vers la fin que

\section{This ys on of Brytayne layes}

That was used by olde dayes.

Est-il vraisemblable que l'original français se fût déjà donné pour un lai breton? Il ne nous est resté aucun poeme français du $\mathrm{XIV}^{e}$ siècle, contenant un récit d'aventure, qui ait été appelé lai par son auteur. ${ }^{3}$ S'agirait-il d'un poème du XIII ${ }^{e}$ siècle dans le genre d'Havelok? C'est possible. Mais il est plus probable que l'auteur d'Emare se borne ici à imiter Chaucer. 4 Avec Sir Gowther, ${ }^{5}$ nous arrivons au $\mathrm{XV}^{e}$ siècle. L'auteur, à ce qu'il nous dit, 6 fonde son poème sur un lai de Bretagne qu'il a longtemps recherché et enfin trouvé. Il se réfère par là évidemment à sa suurce française.

Guigemar est un lai breton et sans doute trois ou quatre autres contes de Marie; si l'on veut désigner par là - et il semble bien que ce soit l'emploi courant du mot - un certain genre littéraire, plus ou moins modelé sur le type de Guigemar, (et à qui ne convient, en dernière analyse, ni le nom de 'lai', ni l'épithète de 'brtton') le conte du Franklin est certainement un spécimen du genre.

I Il est clair qu'on ne se place ici qu'au point de vue de la forme de ces récits: ll s'agit de l'évolution du genre 'lai breton'. (Voir la note précédente.) Les Deux Amants marquent une première étape: une légende, selon toute apparence nullement celtique, nous est contée sous forme de 'lai breton', à l'imitation de Guigemar, et cela par l'autear méme de Guigemar. Doon et Tyolet nous montrent les imitateurs de Maric à l'œuvre: l'esprit leur échappe, mais ils s'assimilent fort bien les procédés, qui du reste leur imposent encore. A vec Renaut nous touchons à la fin de l'évolution: Jui aussi, il sait admirable. ment mettre en œuvre les procédés, mais il s'en moque: il a à cœur de nous montrer qu'il n'est pas dupe. Chaucer est très voisin de l'auteur d'Ignaure: il a aussi alertement pénétré le procédé, il est aussi conscient, mais son tact d'artiste est plus fin: il a senti que le lai breton ne saurait, sans profanation, fournir matière aux plaisanteries. C'est à peine si l'on devine derrière les vers de son prologue un léger sourire.

2 Ed. A. B. Gough, Heidelberg, 1901.

3. l'exception de trois poèmes de Jean de Condé. Cf. Zeitschr.f. rom. Phil., XXIX, 312, n. 1.

- Remarquer la rime layes: dayes qui est dans Chaucer, et dejà dans Sir Orfeo. Elle se retrouve aussi dans l'Ureisun of Ure Lefdi, mais elle $y$ est amenée d'une façon assez différente.

- Ed. Karl Breul, Oppeln, 1886.

B "A lai of Breyten long y sozht | And owt perof a tale have brozht." (v. 29-30). 
Etait-ce vraiment un lai breton? 1 Dans ce cas, il ne serait pas impossible que ce lai fut $T y$ dorel. ${ }^{2}$ Le Comte de Toulouse, 3 également traduit du français, se termine par une strophe où on lit ces vers :

1213 Yn Rome thys geste ys cronyculyd, y wys,

A lai of Bretayne callyd hyt ys,

And evyr more schall bee.

Il est fort douteux que le mot 'lai' fût employé dans l'original français: on n'eût pas ainsi accouplé Rome et Bretagne. Notre traducteur a l'air d'employer ici une expression toute faite que la langue et la tradition lui fournissaient, mais dont il ne perçoit plus nettement le sens. 4 Evidemment, nous touchons ici à la fin. Et en effet, le lai breton a vécu: pendant près de trois siècles, il n'en sera plus question. ${ }^{5}$

Résumons tout ce qui précède. Le mot 'lai' entre en anglais dans le courant du XIIle siècle et, comme il est naturel, il y conserve le sens qu'ıl avait en français, celui de chanson et, en particulier, de chant des oiseaux. La langue avait déjà le mot song, et à l'origine lai, qui n'en était qu'un synonyme un peu plus relevé, dut être d'un emploi assez rare. 6 Mais les traductions ou

1 Remarquer qu'à la différence d'Emare et du Comte de Toulouse Sir Gowther mentionne un lai breton à la fois dans le prologue (v. 29) et dans l'épilogue (v. 753). Ceci est plus conforme à la tradition du 'genre'. (Chaucer n'a pas l'épilogue traditionnel, mais il ne traduit pas du français). Dans Emare le mot 'lai' ne se trouve que dans l'épilogue (v. 1030). De mème dans le Comte de Toulouse (v. 12I4).

2 Voir F. L. Ravenel, Publ. of Mod. Lang. Assoc. of America. XX, 152 et $\mathrm{s}$.

8 Ed. G. Lüdtke, Berlin, 188 I.

- Sens du reste tout conventionnel, comme nous l'avons montré.

- L'expression 'lai breton' et le sens de lai=poème narratif reparaitront vers la fin du XVIII ème siècle, au moment où en France et en Angleterre les érudits et les antiquaires commenceront a explorer les manuscrits du moyenàge. Il y a là une dernière phase de l'histoure du lai (le mot plutôt que le genre, cette fois), qui a son intérét et à laquelle nous nous proposons de consacrer un article spécial.

- Voyez comment, à un peu plus d'un siècle d'intervalle, deux traducteurs ou imitateurs de Wace rendent le passage bien connu où l'auteur de Brut nous décrit les merveilleux talents musicaux de Blégabres, le dieu des jongleurs. (Brut, Ed. le Roux de Lincy, I, p. 178-79, v. 3760-3775). Layamon, au commencement du XIJI ème siècle, rend le passage très fidelement et n'oublie ni la lire et le 'saltérion' ni la harpe et le 'choron'; il est un vers pourtant qu'il laisse de côté: „et mult sot de lais et de note.“ (Ed. F. Madden, I, p. 298-299, v. 6997-70 I2). Vers la fin du premier tiers du siècle suivant, Robert Manning a, dans sa Chronique, l'occasion de traduire le même passage: et, quoiqu'il suive son texte d'assez près, il est plusieurs vers qu'il omet dans sa version: mais s'il néglige de mentionner les instruments aux noms bizarres dont jouait Bleludo, il n'a garde d'oublier les lais: "De note he coupe of alle layes." (The Chronicle of Robert Neanning of Brunne, Rolls Series, 1887, v. 4019-4038). - Ainsi au temps de Layamon, 
adaptations du français, qui deviennent de plus en plus fréquentes, vont multiplier les exemples du mot, et pendant longtemps renforcer l'acception déjà reçue. Il est à noter, du reste. qu'en France la signification du mot s'était quelque peu modifiée au cours des siècles, ou plutôt qu'il n'éveillait plus les mêmes associations d'idées qu'autrefois. Quand il pénètre en français dans la première moitié du $\mathrm{XII}^{e}$ siècle, il retient quelque chose de son origine celtique. Lai, c'est un air ou une chanson qui se chante sur un air breton. Les allusions aux 'lais bretons' sont innombrables dans les auteurs du XII ${ }^{e}$ siècle $:^{1}$ elles sont encore fréquentes dans la première moitié du XIIIe siècle, puis elles se font plus rares et disparaissent. La mélodie ou le rythme que désigne le mot 'lai' se distinguent encore nettement des autres mélodies et des autres rythmes, mais les Bretons n'ont plus rien à $y$ voir: on s'est si bien approprié leurs airs ou on a si bien réussi à en faire de semblables qu'on les a oubliés eux-mêmes. C'est par cette évolution que nous arrivons au lai de Machaut et de Froissart. Elle doit être en grande partie accomplie déjà quand le mot passe en Angleterre, car on ne voit pas qu'il s'y attache, de l'autre côté de la Manche, une nuance de sens qui fasse, en aucune façon, songer à une origine celtique. On nous parle bien des lais, mais non pas des lais bretons. Pour trouver cette expression, il faut arriver aux poèmes de Marie ou à ceux qu'elle a inspirés: ${ }^{2}$ c'est elle qui semble avoir appris aux Anglais l'existence des lais chantés sur la harpe par les Bretons d'autrefois. Même l'auteur du Sir Tristrem, traduisant le Tristan français, ne semble pas s'apercevoir qu'il $y$ ait aucune attache spéciale entre le lai et les Bretons: il emploie le mot de la même facon qu'il emploie song et glee, et comme un synonyme parfois commode à la rime. ${ }^{3}$ A l'occasion, il lui donne un sens si général qu'on ne voit pas comment ailleurs il pourrait $y$ avoir place pour une nuance qui en restreindrait si fort la signification.4 Quoi qu'il en soit, une chose est certaine: à l'origine et pendant assez longtemps, en Angleterre comme en France, le mot implique nécessairement une idée de rythme, de

l'anglais ignore le mot lai: à l'époque de Robert Manning, il lui a donné droit de cite. Entre ces deux dates se place le moment où le mot entre dans la langue et pénètre lentement dans le vocabulaire courant des poètes. Les exemples en sont assez rares alors.

1 Il s'agit ici bien entendu des lais bretons lyriques. Les allusions à des lais bretons narratifs, qu'on ne trouve du reste que chez Marie ou postérieurement à elle, sont au contraires extrêmement rares.

I Il s'agit ici des versions anglaises, le Lay Le Freine par exemple ou Sir Orfeo.

3 Voir Ed. Kölbing, v. 1189-1192: „In his schip was pat day $\mid \mathrm{Al}$ maner of gle | And al maner of lay, | In lond pat mipt be." - Des cinq exemples du mot lai qu'offre le Sir Tristrem, quatre se trouvent à la rime.

4 Ed. Kölbing, v. 1283-1285: ,Ysonde be dede vnderstand, | What alle playes were | In lay." "Allgemeiner für musik", dit le lexique, et dans la traduction le passage est ainsi rendu: "Ysonde lehrte er, wie alle unterhaltenden stücke in der musik wären." 
mélodie, de chant: qui dit lai, dit chanson. C'est seulement dans le premier tiers du XIVe siècle, avec les traductions de Marie et surtout Sir Orfeo, qu'un autre sens - celui de poème narratif sur un sujet celtique - se fait jour d'une façon assez incertaine et hésitante. Chaucer a connaissance de ce nouveau sens, et s'en empare. Il voit bien que le même mot sert à désigner deux choses différentes. Mais la difficulté ne l'embarrasse pas longtemps. Cette confusion, amenée peu à peu par la fantaisie des uns et l'ignorance des autres, il en attribue tout simplement l'origine aux anciens Bretons eux-mêmes. Chansons dites sur la harpe, poèmes faits pour la lecture, ce sont eux qui ont appelé tout cela des lais. Et voilà Chaucer qui, gravement, va nous raconter un lai breton - un de ceux faits pour la lecture. Le lai du Franklin et son ingénieux prologue ont dû trouver plus d'un lecteur intéressé et plus d'un admirateur parmi les comtemporains ou les successeurs immédiats de Chaucer. N'est-ce pas un écho que nous en retrouvons, sinon dans Sir Gowther, du moins dans Emare et le Comte de Toulouse? Mais, même chez Chaucer, le passage est isolé. Nous ne serons donc pas surpris de voir, moins de cinquante ans après lui, disparaître ce sens tout factice d'un vieux mot. - Si l'on accepte ces conclusions, il faut rectifier dans le New English Dictionary l'historique du mot 'lai': „Du XVIe au XVIII ${ }^{e}$ siècle, y lisons-nous, le mot n'est qu'un synonyme poétique de chanson." I Ceci est vrai, sans doute, mais s'applique tout aussi bien aux trois siècles qui précèdent: c'est du XIII ${ }^{e}$ au $\mathrm{XVIII}^{e}$ siècle qu'il fallait dire. L'autre sens, loin d'être le sens original, 2 est dérivé, accidentel; il n'est jamais entré pleinement dans la langue; les exemples en sont très rares, ${ }^{3}$ et il se trouvent tous dans des œuvres qui, plus ou moins directement, se rattachent à Marie, la créatrice de ce néologisme. L'histoire du lai narratif - Le mot et le genre -- est la mème en Angleterre qu'en France, mais, parmi les successeurs français de Marie, il n'y a pas eu de Chaucer.

1 "From the 16 th. to the 18 th c. the word was a mere poetical synonym for "song'."

${ }^{2}$ "Originally applied spec. to the poems, usually dealing with matter of history or romantic adventure, which were sung by minstrels." N. E. D., sv. lay.

${ }_{8}$ Je me suis du reste borné, comme on a pu le voir, à rassembler les exemples cités par les dictionnaires ou dans les travaux de mes prédécesseurs: le nombre ne semble pas, depuis longtemps, s'en être accru.

Lucien Foulet. 